\title{
Two Time Delay Quarantine Model for the Transmission of Worms in Wireless Network
}

\author{
Santosh Kumar Srivastava ${ }^{1}$, Bimal Kumar Mishra ${ }^{2 *}$ and Binay Kumar Mishra ${ }^{3}$ \\ ${ }^{1}$ Department of Computer Applications, Vinoba Bhave University, \\ Hazaribag, India \\ ${ }^{2}$ Department of Mathematics, Birla Institute of Technology Mesra, Ranchi, India \\ ${ }^{3}$ Department of Physics, Veer Kunwar Singh University, Ara, India \\ ${ }^{1}$ srivastava_santosh2@rediffmail.com, ${ }^{2}$ drbimalmishra@gmail.com, \\ 3rbinaymishra@gmail.com
}

\begin{abstract}
A compartmental epidemic model SIQRS with two time delays is developed to understand the attacking behaviour of worms in wireless network. The defence mechanism in wireless network is too weak and is always prone to the attack of worms, specifically, Mabir and Cabir worms. Quarantine compartment is used in this model to fix up the worms attack in the sensor node. Two Time Delays are used to properly understand the time lag of the transmission from one compartment to another in wireless network. Effect of Quarantine is critically analysed. Numerical simulations are performed with examples to validate the analytic results.
\end{abstract}

Keywords: latent time delay, immune time delay, stability, worms, wireless network

\section{Introduction}

Several mathematical models have been formulated, analyzed and applied to a variety of infectious diseases. Mathematical models have become important tools in analyzing the spread and control of infectious diseases. The growth of Internet technology has several classical epidemic model challenges for cyber defense system to safeguard the valuable information stored on the system based on Kermack and McKendrick [1-2]. The spreading behavior of malicious codes in networks is scrutinized by using different epidemiological models.

The two most destructive worms Cabir and Mabir Worms in wireless network are a serious concern these days. Cabir worm display the message "caribe" when a phone is infected via Bluetooth signals till the phone is turned on. Its nature is to create a .sis file but does not spread if the user does not accept the file transfer or does not agree with the installation. Mabir is capable of spreading not only via bluetooth but also via MMS. By sending out copies of itself as a .sis file over cellular networks, it can affect users who are outside the $10 \mathrm{~m}$ range of Bluetooth. As quarantine is one of the important remedial processes for malware attack in network, several researchers developed model taking quarantine as one of the compartment in the epidemic models [3-7]. The effect of time delay on wireless network with worm propagation helps us to better understand the time lag of attack from one compartment to another[8,9].In this paper, we try to develop a compartment susceptible -infectious-quarantine-recover-susceptible(SIQRS) model with emphasis on Two Time Delays : latent and immune period. This model studies the behaviors of virus propagation in the presence of antivirus countermeasures, which are essential for understanding the behavior of worm propagation.

Received (January 1, 2017), Review Result (August 16, 2017), Accepted (September 18, 2017) 


\section{Basic Terminologies}

\section{Susceptible Class}

In this class the nodes are not infected at any point of time t. But nodes are susceptible towards the attack of the malicious signals in the network.

\section{Infectious Class}

In this class, the nodes are infected and are capable to spread the malicious signals to other nodes. The highly infectious nodes are quarantined (isolated) from the network and proper treatment is given to them.

\section{Quarantine Class}

Quarantine is one of the common measures to control or to reduce transmissions of diseases.

\section{Recovered Class}

In this class the infected nodes are recovered either temporarily or permanently. Some nodes lose immunity and return back to the susceptible class.

\section{Delayed Epidemic Model Formulation}

Our model is based on SIQRS model which has Susceptible, Infectious, Quarantine and Recovered classes. Our assumptions are as follows:-

a) The total population of nodes is divided into four compartments: Susceptible, Infectious, Quarantine and Recovered nodes respectively represented as $S(t), I(t), Q(t)$ and $\mathrm{R}(\mathrm{t})$ respectively.

b) Susceptible nodes become infected but it does not move to the Quarantine class due to low energy. This type of node remains in the infected class for a certain latent period $\tau_{1}$ before quarantine.

c) $\beta$ is the Infectivity Contact Rate from Susceptible class to Infectious class. Let $d_{1}$ be the natural death rate and $d_{2}$ be the crashing of the nodes due to infectious worms.

d) The number of nodes transfer from Infectious class to Quarantine class with transmission rate $\epsilon . \gamma$ and $\alpha$ are the rate at which nodes get transferred to Recovered class from Infected and Quarantine classes respectively.

e) Infectious nodes get recovered and moves to susceptible class at the rate of $\delta$.

The schematic diagram for the flow of worms in sensor network is depicted in Figure 1. Based on the flow of attack and assumptions, we have the following system of equations:

$$
\begin{aligned}
& \frac{d S}{d t}=A-\mathrm{d}_{1} \mathrm{~S}(\mathrm{t})-\beta \mathrm{S}(\mathrm{t}) \mathrm{I}(\mathrm{t})+\delta R(t) \\
& \frac{d I}{d t}=\beta e^{-\mathrm{d}_{1} \tau_{1} \mathrm{~S}\left(\mathrm{t}-\tau_{1}\right) \mathrm{I}\left(\mathrm{t}-\tau_{1}\right)-\left(\mathrm{d}_{1}+\mathrm{d}_{2}+\gamma+\epsilon\right) \mathrm{I}(\mathrm{t})} \\
& \frac{d Q}{d t}=\epsilon I(\mathrm{t})-\left(\mathrm{d}_{1}+\mathrm{d}_{2}\right) \mathrm{Q}(\mathrm{t})-\alpha \mathrm{Q}(\mathrm{t}) \\
& \frac{d R}{d t}=\alpha Q(\mathrm{t})-\mathrm{d}_{1} \mathrm{R}(\mathrm{t})+\gamma \mathrm{I}(\mathrm{t})-\delta R(t)
\end{aligned}
$$

where $\tau_{1} \geq 0$ represents a time delay describing the latent period of infected nodes, the term $\beta e^{-\mathrm{d}_{1} \tau_{1}} \mathrm{~S}\left(\mathrm{t}-\tau_{1}\right) \mathrm{I}\left(\mathrm{t}-\tau_{1}\right)$ represents the nodes surviving in the latent period $\tau_{1}$ and becoming infective at time $t$, and $\mathrm{A}$ be the total input rate of node . 


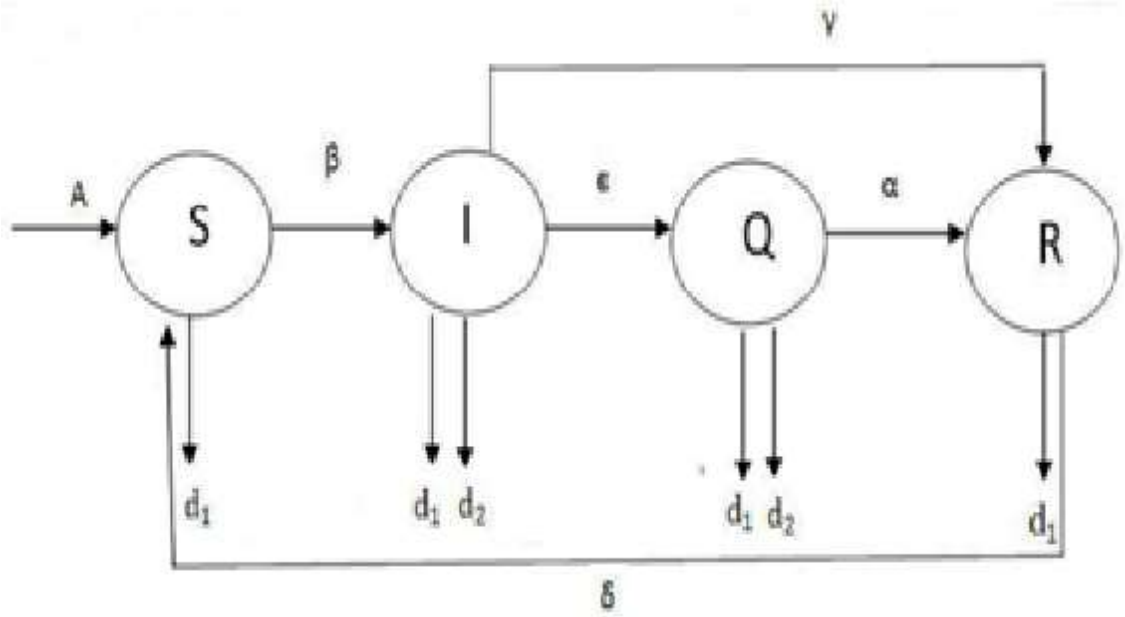

Figure 1. Schematic Diagram of SIQRS

Let there be a fixed duration of temporary immunity, after which the Recovered nodes return to the class of Susceptible. The temporary immunity is incorporated in our model by the term $\mathrm{R}\left(\mathrm{t}-\tau_{2}\right) e^{-\mathrm{d}_{1} \tau_{2}}$, where $\tau_{2}$ is the length of immunity period. This term reflects the fact that the node has survived from the natural death in a recovery pool before becoming Susceptible again.

Incorporating this time delay $\tau_{2}$ in (1), we have the modified model as:

$$
\begin{gathered}
\frac{d S}{d t}=A-\mathrm{d}_{1} \mathrm{~S}(\mathrm{t})-\beta \mathrm{S}(\mathrm{t}) \mathrm{I}(\mathrm{t})+\delta R\left(\mathrm{t}-\tau_{2}\right) e^{-\mathrm{d}_{1} \tau_{2}} \\
\frac{d I}{d t}=\beta e^{-\mathrm{d}_{1} \tau_{1}} \mathrm{~S}\left(\mathrm{t}-\tau_{1}\right) \mathrm{I}\left(\mathrm{t}-\tau_{1}\right)-\left(\mathrm{d}_{1}+\mathrm{d}_{2}+\gamma+\epsilon\right) \mathrm{I}(\mathrm{t}) \\
\frac{d Q}{d t}=\epsilon I(\mathrm{t})-\left(\mathrm{d}_{1}+\mathrm{d}_{2}\right) \mathrm{Q}(\mathrm{t})-\alpha \mathrm{Q}(\mathrm{t}) \\
\frac{d R}{d t}=\alpha Q(\mathrm{t})-\mathrm{d}_{1} \mathrm{R}(\mathrm{t})+\gamma \mathrm{I}(\mathrm{t})-\delta R\left(\mathrm{t}-\tau_{2}\right) e^{-\mathrm{d}_{1} \tau_{2}}
\end{gathered}
$$

Where $\delta$ is the rate at which Recovered nodes lose immunity and return to the Susceptible class and all the parameters are positive constants.

The initial condition of (2) is given as

$$
\begin{gathered}
\mathrm{S}(\theta)=\phi_{1}(\theta), \mathrm{S}(\theta)=\phi_{2}(\theta), \mathrm{S}(\theta)=\phi_{3}(\theta), \mathrm{S}(\theta)=\phi_{4}(\theta) \\
\phi_{1}(\theta)>0, \theta \in[-\tau, 0], \phi_{1}(\theta) \geq 0, \tau=\max \left[\tau_{1}, \tau_{2}\right]
\end{gathered}
$$

$\phi_{\mathrm{i}}(\theta)>0, \theta \in[-\tau, 0], \phi_{\mathrm{i}}(\theta)>0, i=2,3,4$ where $\tau=\max \left[\tau_{1}, \tau_{2}\right]$

Where $\left(\phi_{1}(\theta), \phi_{2}(\theta), \phi_{3}(\theta), \phi_{4}(\theta)\right) \in c\left([-\tau, 0], \mathrm{R}_{+\theta}^{4}\right)$

The Banach Space of continuous functions, mapping the interval $[-\tau, 0]$ into $R_{+\theta}^{4}$ ,where

$$
\mathrm{R}_{\theta}^{4}=\left\{\left(\mathrm{x}_{1}, \mathrm{x}_{2}, \mathrm{x}_{3}, \mathrm{x}_{4}\right): \mathrm{x}_{1}>0, \mathrm{x}_{\mathrm{i}} \geq 0, \mathrm{i}=2,3,4\right\}
$$

For continuity of the initial condition, we have

$$
\mathrm{E}(\theta)=\int_{-\tau}^{0} \beta e^{\mathrm{d}_{1} \theta} \varphi_{1}(\theta) \varphi_{2}(\theta) \mathrm{d} \theta
$$

It is well known from the fundamental theory of Functional Equation [10] that system (2) has a unique solution $(S(t), I(t), Q(t), R(t))$ satisfying the initial conditions (3) and (4). It is easy to show that all solutions of the system (2) with initial conditions (3) and (4) are defined on $[0,+\infty)$ and remain positive for all $t \geq 0$ 


\section{Equilibrium Points and Threshold Number}

Equilibrium points show the different kinds of behaviour in the long run of the system. It provides us mathematical conditions based on which the long term behaviour of the network can be predicted and classified into a finite number of possibilities.

$$
\begin{aligned}
\frac{d S}{d t}= & A-\mathrm{d}_{1} \mathrm{~S}(\mathrm{t})-\beta \mathrm{S}(\mathrm{t}) \mathrm{I}(\mathrm{t})+\delta R\left(\mathrm{t}-\tau_{2}\right) e^{-\mathrm{d}_{1} \tau_{2}} \\
& \frac{d I}{d t}=\beta e^{-\mathrm{d}_{1} \tau_{1}} \mathrm{~S}\left(\mathrm{t}-\tau_{1}\right) \mathrm{I}\left(\mathrm{t}-\tau_{1}\right)-\left(\mathrm{d}_{1}+\mathrm{d}_{2}+\gamma+\epsilon\right) \mathrm{I}(\mathrm{t}) \\
\frac{d Q}{d t}= & \epsilon I(\mathrm{t})-\left(\mathrm{d}_{1}+\mathrm{d}_{2}\right) \mathrm{Q}(\mathrm{t})-\alpha \mathrm{Q}(\mathrm{t}) \\
\frac{d R}{d t}= & \alpha Q(\mathrm{t})-\mathrm{d}_{1} \mathrm{R}(\mathrm{t})+\gamma \mathrm{I}(\mathrm{t})-\delta R\left(\mathrm{t}-\tau_{2}\right) e^{-\mathrm{d}_{1} \tau_{2}}
\end{aligned}
$$

For equilibrium points, we have

$$
\begin{aligned}
& \frac{d S}{d t}=0 ; \frac{d I}{d t}=0 ; \frac{d Q}{d t}=0 ; \frac{d R}{d t}=0 ; \\
& A-\mathrm{d}_{1} \mathrm{~S}(\mathrm{t})-\beta \mathrm{S}(\mathrm{t}) \mathrm{I}(\mathrm{t})+\delta R\left(\mathrm{t}-\tau_{2}\right) e^{-\mathrm{d}_{1} \tau_{2}}=0
\end{aligned}
$$

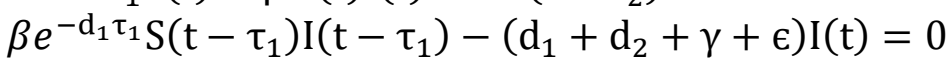

$$
\begin{aligned}
& \epsilon I(\mathrm{t})-\left(\mathrm{d}_{1}+\mathrm{d}_{2}\right) \mathrm{Q}(\mathrm{t})-\alpha \mathrm{Q}(\mathrm{t})=0 \\
& \alpha Q(\mathrm{t})-\mathrm{d}_{1} \mathrm{R}(\mathrm{t})+\gamma \mathrm{I}(\mathrm{t})-\delta R\left(\mathrm{t}-\tau_{2}\right) e^{-\mathrm{d}_{1} \tau_{2}}=0
\end{aligned}
$$

the worm free-equilibrium $\mathrm{E}_{1}\left(\mathrm{~S}_{0}, \mathrm{I}_{0}, \mathrm{Q}_{0}\right)=\left(\frac{A}{\mathrm{~d}_{1}}, 0,0\right)$ and endemic equlibrium is $\mathrm{E}_{2}\left(\mathrm{~S}^{*}, I^{*}, Q^{*}\right)$,where,

$$
\begin{aligned}
& \mathrm{S}^{*}=\frac{\mathrm{d}_{1}+\mathrm{d}_{2}+\gamma+\epsilon}{\beta e^{-\mathrm{d}_{1} \tau_{1}}} \\
& I^{*} \\
& =\frac{\left(\mathrm{d}_{1}\left(\mathrm{~d}_{1}+\mathrm{d}_{2}+\gamma+\epsilon\right)-\mathrm{A} \beta e^{-\mathrm{d}_{1} \tau_{1}}\right)\left(\alpha \epsilon+\gamma\left(\mathrm{d}_{1}+\mathrm{d}_{2}+\alpha\right)\right)\left(\mathrm{d}_{1}+\delta e^{-\mathrm{d}_{1} \tau_{2}}\right)\left(\mathrm{d}_{1}+\mathrm{d}_{2}+\alpha\right)}{\left(\left(\mathrm{d}_{1}+\mathrm{d}_{2}+\gamma+\epsilon\right)\left(\mathrm{d}_{1} \delta e^{-\mathrm{d}_{1} \tau_{2}}\right)\left(\mathrm{d}_{1}+\mathrm{d}_{2}+\alpha\right)-\delta e^{-\mathrm{d}_{1} \tau_{1}} e^{-\mathrm{d}_{1} \tau_{2}}\left(\alpha \epsilon+\gamma\left(\mathrm{d}_{1}+\mathrm{d}_{2}+\alpha\right)\right)\right)\left(\alpha \epsilon+\gamma\left(\mathrm{d}_{1}+\mathrm{d}_{2}+\alpha\right)\right)} \\
& Q^{*} \\
& =\frac{\epsilon\left(\mathrm{d}_{1}\left(\mathrm{~d}_{1}+\mathrm{d}_{2}+\gamma+\epsilon\right)-\mathrm{A} \beta e^{-\mathrm{d}_{1} \tau_{1}}\right)\left(\alpha \epsilon+\gamma\left(\mathrm{d}_{1}+\mathrm{d}_{2}+\alpha\right)\right)\left(\mathrm{d}_{1}+\delta e^{-\mathrm{d}_{1} \tau_{2}}\right)}{\left(\left(\mathrm{d}_{1}+\mathrm{d}_{2}+\gamma+\epsilon\right)\left(\mathrm{d}_{1} \delta e^{-\mathrm{d}_{1} \tau_{2}}\right)\left(\mathrm{d}_{1}+\mathrm{d}_{2}+\alpha\right)-\delta e^{-\mathrm{d}_{1} \tau_{1}} e^{-\mathrm{d}_{1} \tau_{2}}\left(\alpha \epsilon+\gamma\left(\mathrm{d}_{1}+\mathrm{d}_{2}+\alpha\right)\right)\right)\left(\alpha \epsilon+\gamma\left(\mathrm{d}_{1}+\mathrm{d}_{2}+\alpha\right)\right)} \\
& R^{*}=\frac{\left(\mathrm{d}_{1}\left(\mathrm{~d}_{1}+\mathrm{d}_{2}+\gamma+\epsilon\right)-\mathrm{A} \beta e^{-\mathrm{d}_{1} \tau_{1}}\right)\left(\alpha \epsilon+\gamma\left(\mathrm{d}_{1}+\mathrm{d}_{2}+\alpha\right)\right)}{\left(\mathrm{d}_{1}+\mathrm{d}_{2}+\gamma+\epsilon\right)\left(\mathrm{d}_{1} \delta e^{-\mathrm{d}_{1} \tau_{2}}\right)\left(\mathrm{d}_{1}+\mathrm{d}_{2}+\alpha\right)-\delta e^{-\mathrm{d}_{1} \tau_{1}} e^{-\mathrm{d}_{1} \tau_{2}}\left(\alpha \epsilon+\gamma\left(\mathrm{d}_{1}+\mathrm{d}_{2}+\alpha\right)\right)}
\end{aligned}
$$

The Basic Reproduction Number denoted by $R_{0}$, is one of the most important parameter of an epidemic. $R_{0}$ expresses the expected number of secondary infection generated by a single infectious node into a fully susceptible collection of nodes.

Form system (2), we get

$$
\begin{aligned}
& \mathrm{I}^{\prime}(t)=\beta e^{-\mathrm{d}_{1} \tau_{1}} \mathrm{~S}\left(\mathrm{t}-\tau_{1}\right) \mathrm{I}\left(\mathrm{t}-\tau_{1}\right)-\left(\mathrm{d}_{1}+\mathrm{d}_{2}+\gamma+\epsilon\right) \mathrm{I}(\mathrm{t}) \\
& \mathrm{I}^{\prime}(t) \leq \beta e^{-\mathrm{d}_{1} \tau_{1}} \mathrm{~S}_{0} \mathrm{I}\left(\mathrm{t}-\tau_{1}\right)-\left(\mathrm{d}_{1}+\mathrm{d}_{2}+\gamma+\epsilon\right) \mathrm{I}(\mathrm{t})
\end{aligned}
$$

Initially, when we introduce an infectious node into the whole collection of Susceptible nodes it will still be approximately $\mathrm{S}_{0}$, until a large number of new secondary infectious nodes are formed. $\mathrm{R}_{0}$ is defined to be the average period of the ratio of the Coefficients of the Upper Bond for $\mathrm{I}(\mathrm{t})$ [11], i.e.

If $\mathrm{I}^{\prime}(t) \leq \mathrm{a}(\mathrm{t}) \mathrm{I}\left(\mathrm{t}-\tau_{1}\right)-\mathrm{b}(\mathrm{t}) \mathrm{I}(\mathrm{t})$ for positive valued functions $\mathrm{a}(\mathrm{t})$ and $\mathrm{b}(\mathrm{t})$, then

We have ,

$$
\mathrm{R}_{0}=\frac{1}{\tau} \int_{0}^{\tau} \frac{\mathrm{a}(\mathrm{t})}{\mathrm{b}(\mathrm{t})} d t
$$

$$
\mathrm{R}_{0}=\frac{1}{\tau} \int_{0}^{\tau} \frac{\beta e^{-\mathrm{d}_{1} \tau_{1}} \mathrm{~S}_{0}}{\left(\mathrm{~d}_{1}+\mathrm{d}_{2}+\gamma+\epsilon\right)} d t
$$


Or $\quad \mathrm{R}_{0}=\frac{\beta e^{-\mathrm{d}_{1} \tau_{1}} A}{\mathrm{~d}_{1}\left(\mathrm{~d}_{1}+\mathrm{d}_{2}+\gamma+\epsilon\right)}$

\section{Analysis and Stability of the System}

\subsection{Local Stability of the System}

Theorem 1: If $\mathrm{R}_{0}<1$, then the worm free equilibrium $\mathrm{E}_{1}$ is locally asymptotically stable and unstable if $\mathrm{R}_{0}>1$.

Proof: The characteristics of the Linearization of System (5) near the worm free equilibrium $\mathrm{E}_{1}$ is given by

$$
\left(-d_{1}-\gamma\right)\left(-d_{1}-d_{2}-\gamma-\lambda-\epsilon\right)\left(-d_{1}-d_{2}-\alpha-\lambda\right)=0
$$

Here equation (8) always has negative real root

$\lambda_{1}=-\mathrm{d}_{1}, \lambda_{2}=-\left(\mathrm{d}_{1}+\mathrm{d}_{2}+\alpha\right)$ and $\lambda_{3}=-\left(\mathrm{d}_{1}+\mathrm{d}_{2}+\gamma+\epsilon\right)$

As all the roots are negative, so worm free equilibrium is locally asymptotically stable.

\subsection{Global Stability of the System}

Let us consider the following equation

$$
\mathrm{U}(\mathrm{t})=a \mathrm{U}(\mathrm{t}-\tau)-\mathrm{bU}(\mathrm{t})-\mathrm{cU}^{2}(\mathrm{t})
$$

Where $a, b$ and $c$ are positive constants $\geq 0$, equation (9) always has a trivial equilibrium $\mathrm{U}_{0}=0$.If $a>b$,then equation (9) has a unique positive equilibrium.

$\mathrm{U}^{*}=\frac{a-b}{c}$.

Lemma, If, $a>b$ then the positive equilibrium $\mathrm{U}^{*}=\frac{a-b}{c}$ is globally stable; if $a<b$ then the trivial equilibrium $U_{0}=0$ is globally asymptotically stable.

Proof of the above lemma is very similar to that of lemma 3.1 in Song et.al., [12], we therefore, omit it here.

From the system (9) we take the following transformation:

$$
\mathrm{s}^{\prime}=\frac{a}{d_{1}}-s ; \quad \mathrm{I}^{\prime}=I ; \quad \mathrm{Q}^{\prime}=Q
$$

After this transformation, system (2) becomes:

$$
\begin{gathered}
\frac{d s^{\prime}}{d t}=-d_{1} s(t)+\beta\left(\frac{A}{d_{1}}-s^{\prime}(t)\right) I^{\prime}(t)-s i^{\prime}\left(t-T_{2}\right) e^{-d_{1} T_{2}} \\
\frac{d I^{\prime}}{d t}=\beta e^{-d_{1} T_{1}}\left(\frac{A}{d_{1}}-s^{\prime}\left(t-T_{1}\right)\right) I^{\prime}\left(t-T_{1}\right)-\left(d_{1}+d_{2}+\gamma+\epsilon\right) I^{\prime}(t) \\
\frac{d Q^{\prime}}{d t}=\in I^{\prime}(t)-\left(d_{1}+d_{2}\right) Q^{\prime}(t)-\alpha Q^{\prime}(t)
\end{gathered}
$$

Now the system $(10)$ has an equilibrium $(0,0,0)$. In order to show the global stability of the point $E_{1}$ for the system (5), we have to prove the global stability of the point $(0,0,0)$ for the system (10).

The equation for $I^{\prime}(t)$ can be rewritten as follows

$$
\begin{array}{r}
\frac{d I^{\prime}}{d t}=\beta e^{-d_{1} T_{1}}\left(\frac{A}{d_{1}}-s^{\prime}\left(t-T_{1}\right)\right) I^{1}\left(t-T_{1}\right)-\left(d_{1}+d_{2}+\gamma+\epsilon\right) I^{\prime}(t) \\
\leq\left[\frac{A}{d_{1}} \beta e^{-d_{1} T_{1}} I^{\prime}\left(t-T_{1}\right)\right]-\left(d_{1}+d_{2}+\gamma+\epsilon\right) I^{\prime}(t)
\end{array}
$$

Under the condition $\mathrm{R}_{0}<1$, and by the above mentioned lemma, a comparative argument shows that

$$
I^{\prime}(t) \rightarrow 0,(t \rightarrow \infty)
$$

Now we prove that $Q^{\prime}(t) \rightarrow 0$ as $t \rightarrow \infty$ from the third equation of (10) 
By using (9) we can show that

$$
\frac{d Q^{\prime}}{d t} \leq \in I^{\prime}(t)-\left(d_{1}+d_{2}\right) Q^{\prime}(t)-\alpha Q^{\prime}(t)
$$

$$
\begin{aligned}
& Q^{\prime}(t) \rightarrow 0,(t \rightarrow \infty) \\
& \frac{d S^{\prime}}{d t} \leq d_{1} S^{\prime}(t)+\left(d_{1}+d_{2}+\gamma+\epsilon\right) I^{\prime}\left(t-\tau_{1}\right)+d_{1} Q^{\prime}(t)-\delta R^{\prime}\left(t-\tau_{2}\right) e^{-d_{1} \tau_{2}}
\end{aligned}
$$

From equation (11) and (12), it can be seen that the solution of (13) are bounded by exponentially decaying function as $(t \rightarrow \infty)$, hence the system is globally asymptotically stable at $\mathrm{E}_{1}$.

\section{Numerical Simulations}

Runge-Kutta-Fehlberg method of order 4 and 5 is used to solve and simulate the system (3) using different parametric values.

Example1: Let the system (2) with $\mathrm{A}=.33, \mathrm{~d}_{1}=.39, \quad \beta=.3, \delta=.04$, $\mathrm{d}_{2}=.04, \gamma=.04, \epsilon=.03, \alpha=.02$ be in the Infectious -Recovered plane .We try to understand the nature of the trajectory towards the worm free equilibrium point when $R_{0}<1$.we observe that the system is locally asymptotically table(Figure 2 ).

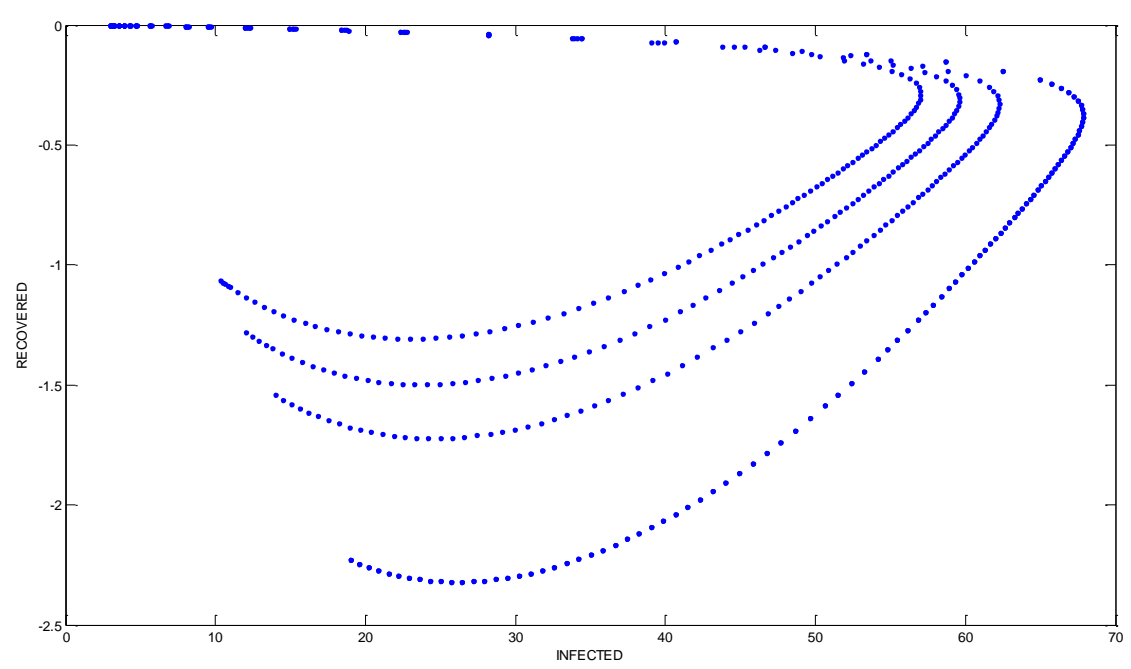

Figure 2. Global Stability of Worm Free Equilibrium Point when $R_{0}<1$

Example 2: Let the system (2) be with $\mathrm{A}=.33, \mathrm{~d}_{1}=.47, \beta=.3, \delta=.04, \mathrm{~d}_{2}=.04, \gamma=.04$, $\epsilon=.03, \alpha=.02$.We see that the different initial points satisfy the condition $R_{0}>1$.We observe that all trajectories asymptotically reaches the point of stability(Figure 3). 


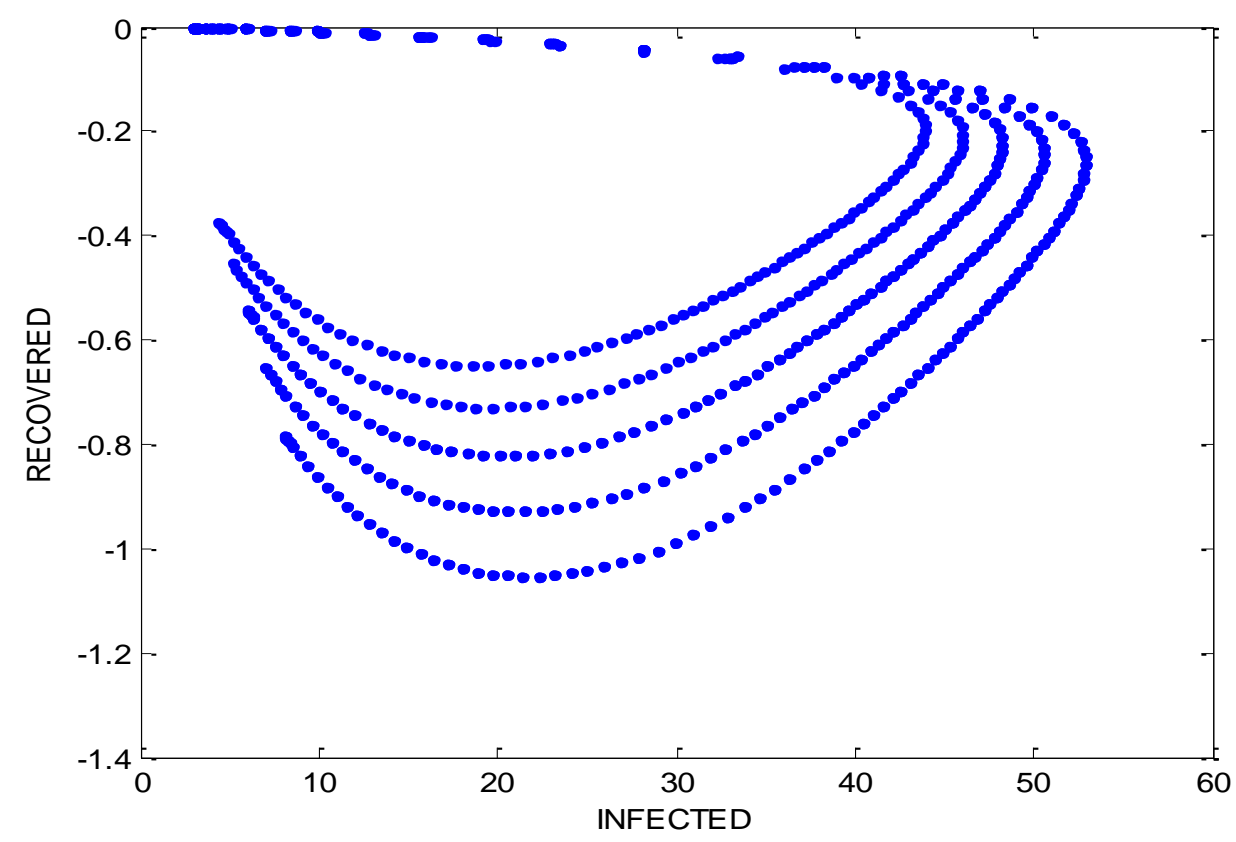

Figure 3. Global Stability of Worm Free Equilibrium Point when $R_{0}>1$

Example 3: Let the system (2) be with $A=.33, \mathrm{~d}_{1}=.39, \beta=.3, \delta=.04, \mathrm{~d}_{2}=.04, \gamma=.04$, $\epsilon=.03, \alpha=.02$.s. Here $\tau_{1}$ and $\tau_{2}$ are Two Time Delay whose different values are given on the legend of the Figure 4. It shows the effect of time delay on Infectious Class. We see that increasing the value of $\tau_{1}$ and keeping $\tau_{2}$ constant, the graph of Infectious Class goes on increasing. Here the time delay increases and the Infectious node decreases (Figure 4).

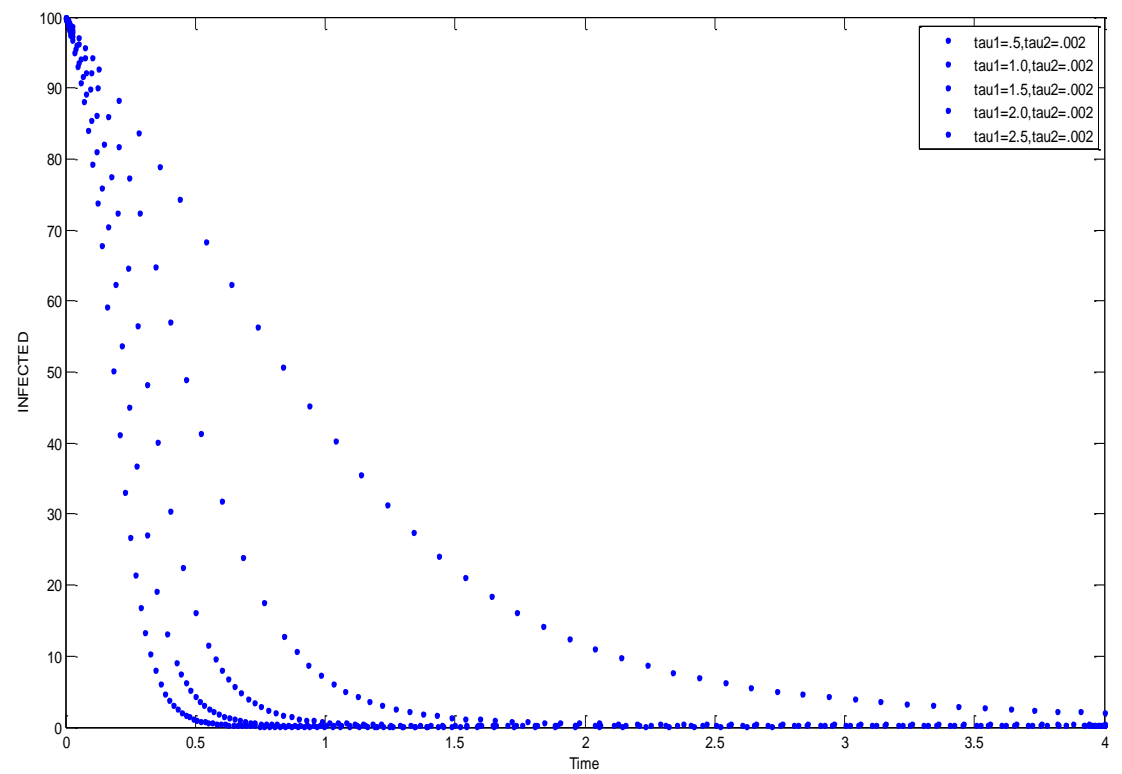

Figure 4. Effect of Delay on Infectious Class

Example 4: Let the system (2) be with $A=.33, \mathrm{~d}_{1}=.39, \beta=.3, \delta=.04, \mathrm{~d}_{2}=.04, \gamma=.04$, $\epsilon=.03, \alpha=.02$. Here we see that due to quarantine compartment worms are recovered more and reaches the point of stability with two time delay (Figure 5). 


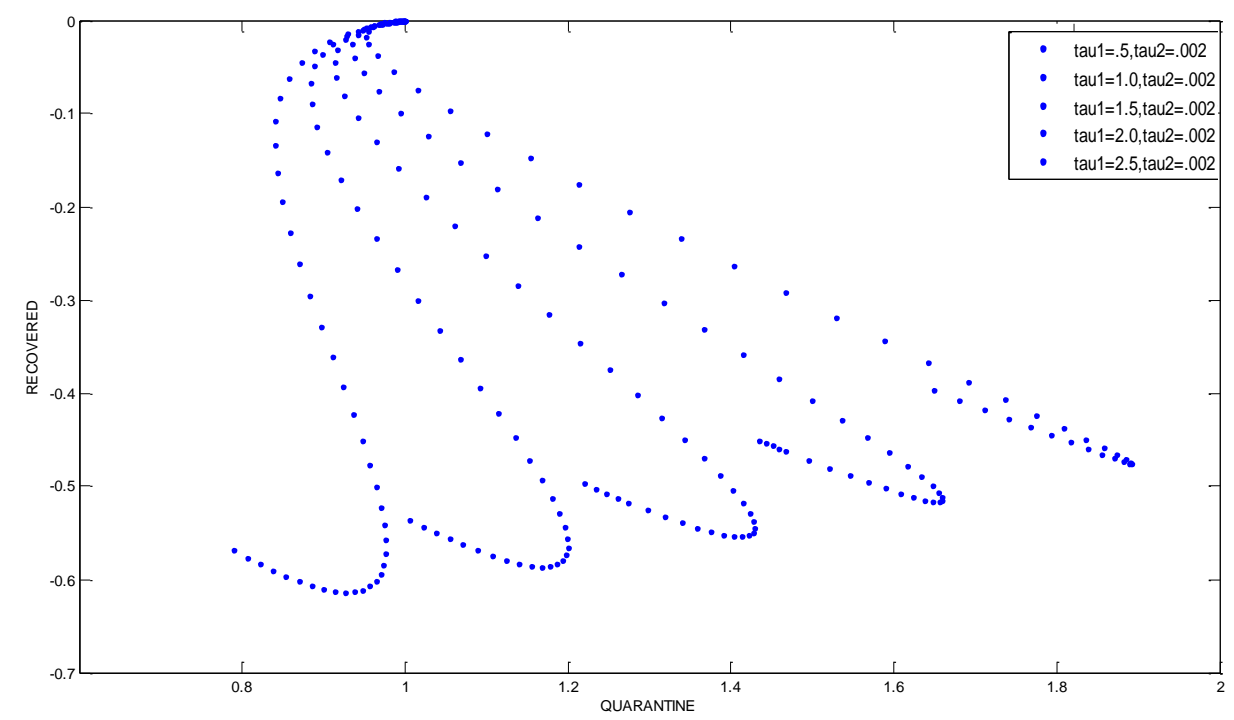

Figure 5. Effect of Delay on Q-R Plane

\section{Conclusion}

A critical analysis on the effect of Latency time delay and Immunity time delay is performed in this paper. We use SIQRS (Susceptible -Infected- Quarantine - Recovered Susceptible) model. We have seen the effect of time delay in different compartment of SIQRS model. We observe that too large delay may tend to large number of infected nodes. We establish the local and global asymptotic stability of the worm free equilibrium point of the system when the Basic Reproduction Number has the value less than one. When $R_{o}$ is greater than one, epidemic starts. The introduction of time delay decreases the possibilities of worm propagation in wireless network. Effect of quarantine is also analyzed and it is observed that: more we quarantine the infectious nodes, the more rapidly we recover.

\section{References}

[1] W.O. Kermack and A.G. Mckendrick, "Contributions of Mathematical Theory to Epidemics", Proc. R. Soc. Lond C Ser, vol. A, no. 115, (1927), pp. 700C721.

[2] W.O. Kermack and A.G Mckendrick, "Contributions of mathematical theory to epidemics", Proc. R. Soc. Lond. C Ser, vol. A, no. 138, (1932), pp. 55C83.

[3] B.K. Mishra and N. Jha, "SEIQRS model for the transmission of malicious objects in Computer Nework", Applied Mathematical Modelling, vol. 34, (2010), pp. 710-715.

[4] D. Moore, C. Shannon, G.M. Voelker and S. Savage, "Internet quarantine: requirements for containing self Cpropagating code", in: Proceedings of IEEE INFOCOM 2003, IEEE, (2003).

[5] P. De, Y. Liu and S.K. Das, "An Epidemic Theoretic Framework for evaluating Broadcast Protocols in Wireless Sensor Networks", in: Proc. IEEE (Intl Conf. on Mobile Adhoc and Sensor Systems (MASS), (Pisa, Italy), October 2007.12Transaction on IoT and Cloud Computing, vol. 2, no. 1, (2014), pp. 1-13.

[6] C.C. Zou, W. Gong and D. Towsley, "Worm propagation modeling and analysis under dynamic quarantine defense", in: Proceedings of the ACM CCSWorkshop on Rapid Malcode, ACM, (2003), pp. 51-60.

[7] B.K. Mishra and A.K. Singh, "Two Quarantine Models in the attack of Malicious Objects in Computer Network", Hindwai Publishing Corporation, Mathematical Problems In Engineering, Article ID 407064, doi:10.1155/2012/407604, (2012), p. 13.

[8] N. Keshri and B.K. Mishra, "Two Time Delay Dynamic Model on the Transmission of Malicious Signals in Wireless Sensor Network", Chaos, Solitons and Fractals, vol. 68, (2014), pp. 151-158. 
[9] T. Dong, X. Liao and H. Li, "Stability and Hopf Bifurcation in a Computer Virus Model with Multistate Antivirus", Abstract and Applied Analysis, Article ID 841987, http://dx.doi.org/10.1155/2012 / 841987, vol. 2012, (2012), p. 16.

[10] J. Hale, "Theory of Functional Differential Equations", Heidelberg Springer, (1977).

[11] L. Nagy, "Epidemic models with pulse vaccination and time delay", Ontario, Canada: University of Waterloo, (2011)

[12] X. Song and L. Chen, Optimal harvesting and stability for a two -species competitive system with stage structure Math Bioscience, vol. 170, (2001), pp. 173-186. 
International Journal of Security and Its Applications

Vol. 11, No. 10 (2017) 